TRANSACTIONS OF THE

AMERICAN MATHEMATICAL SOCIETY

Volume 334, Number 1, November 1992

\title{
$\Sigma_{n}$ DEFINABLE SETS WITHOUT $\Sigma_{n}$ INDUCTION
}

\author{
C. T. CHONG AND K. J. MOURAD
}

\begin{abstract}
We prove that the Friedberg-Muchnik Theorem holds in all models of $\Sigma_{1}$ collection under the base theory $P^{-}+I \Sigma_{0}$. Generalizations to higher dimensional analogs are discussed. We also study the splitting of r.e. sets in these weak models of arithmetic.
\end{abstract}

The study of recursively enumerable (r.e.) sets is one of the focal points of modern recursion theory, with a history going back to the investigations initiated by Post. Post's problem asks whether there exists a nonrecursive incomplete r.e. set over $\omega$. This problem is answered affirmatively by the FriedbergMuchnik Theorem which asserts the existence of two r.e. sets having incomparable Turing degrees. The method of priority argument was introduced for the solution, and since then the technique has been developed and refined to a level of extraordinary sophistication and complexity.

The Friedberg-Muchnik Theorem has been generalized in many directions. Sacks and Simpson [14] proved this for all admissible ordinals, and Friedman [7] obtained it for many inadmissibe ordinals. Also, in [3] we show that a related and natural version of Post's problem has a positive solution for all limit ordinals and that the same holds when relativized to $\varnothing^{\prime}$ for all admissible ordinals. In another direction, Simpson (unpublished) has shown that the priority argument used for the solution holds in all models of fragments of Peano arithmetic satisfying $\Sigma_{1}$ induction, and Slaman and Woodin [15] have shown that in all models of $\Sigma_{1}$ collection there is a positive solution to the original Post problem. The more difficult question of whether the Friedberg-Muchnik Theorem holds in these models was left open.

In this paper we study some basic problems of r.e. sets in the setting of firstorder arithmetic. We work in the usual first-order language of arithmetic with an additional function symbol for exponentiation. Let $P^{-}+I \Sigma_{0}$ denote the set of Peano axioms (including the definition of exponentiation), yet with the full induction scheme replaced by induction restricted to $\Sigma_{0}$ formulas. Let $B \Sigma_{n}$ and $I \Sigma_{n}$ denote respectively the $\Sigma_{n}$ collection and the $\Sigma_{n}$ induction scheme. Paris and Kirby [13] proved that over the base theory $P^{-}+I \Sigma_{0}, I \Sigma_{n}$ implies

Received by the editors September 6, 1990.

1991 Mathematics Subject Classification. Primary 03D99, 03D55.

Key words and phrases. Recursively enumerable sets, Friedberg-Muchnik Theorem, Sacks Splitting Theorem, fragments of Peano arithmetic.

Part of this work was done while the first author was a member of the Mathematical Sciences Research Institute, Berkeley, California, during the period January-June, 1990. The hospitality of the MSRI is gratefully acknowledged. 
$B \Sigma_{n}$, but not conversely, and that $B \Sigma_{n+1}$ implies $I \Sigma_{n}$, but not conversely. Many basic results on r.e. sets and degrees are known to be consequences of systems like $P^{-}+I \Sigma_{1}$ (for finite injury), and $P^{-}+I \Sigma_{2}$ (for infinite injury), and fail to hold for weaker systems (see [11, 12, 15]). Interestingly, some results or strategies are discovered to be equivalent to $I \Sigma_{1}$ or $I \Sigma_{2}$ over a base theory (see [1] for maximal sets, [8] for some finite and infinite strategies, and [10] for the Sacks splitting theorem). Results in the last category may be interpreted as pertaining to the field of reverse recursion theory.

We first prove (Theorem 1 in $\S 1$ ) that in every model (including the model studied in [15]) of $P^{-}+I \Sigma_{0}+B \Sigma_{1}$, the Friedberg-Muchnik Theorem has a positive solution. A corollary is that, proof-theoretically speaking, the Sacks Splitting Theorem is strictly stronger than the Friedberg-Muchnik Theorem.

A natural analog of the Friedberg-Muchnik type problem for higher dimension can be phrased as follows: Given $n>1$, are there $\Sigma_{n} \backslash \Delta_{n}$ sets having incomparable degrees in some (or any) model of $P^{-}+I \Sigma_{0}+B \Sigma_{n}$ ? In addition, we may require that the two sets lie above $\varnothing^{(n-1)}$. Using an oracle approach, one may extend the method of proof in $\S 1$ to derive the higher dimensional analog. In this case, one can obtain two $\Sigma_{n}$ sets above $\varnothing^{(n-1)}$ which are incomparable even by $\Sigma_{n}$ functionals. It is interesting to contrast this question with the weaker one that only asks whether there are two incomparable $\Sigma_{n} \backslash \Delta_{n}$ sets. In this case, one can 'transfer' results about r.e. degrees to $\Sigma_{n}$ degrees. These degrees however are not above the degree of $\varnothing^{(n-1)}$.

In $\S 2$ we discuss these issues. We investigate the structure of the $\Sigma_{n} \backslash \Delta_{n}$ degrees and the $\Sigma_{n}$ degrees above the degree of $\varnothing^{(n-1)}$. Here we use a result from [5] to study the $\Sigma_{n}$ degrees above the degree of a $\Sigma_{n}$ cut which are not above the degree of $\varnothing^{(n-1)}$. The technique employed in [5] is a variant of the one used in [2] to study the minimal pair and the minimal degree problem. The advantage to be gained from this approach is that it establishes a general orderpreserving correspondence, under the join map, between a class of amenable sets (including the $\Delta_{2}$ sets) and a class of $\Sigma_{n}$ sets above a $\Sigma_{n}$ cut, thus pointing to the rich structure of definable sets residing in models of $P^{-}+I \Sigma_{0}+B \Sigma_{n}+\neg I \Sigma_{n}$ above a $\Sigma_{n}$ cut. Such information is not yet available above $\varnothing^{(n-1)}$ because of the difficulties of handling nonamenable sets in these models. We will elaborate on this in the sequel.

Finally, in $\S 3$ we study splitting properties of r.e. sets in models of $B \Sigma_{1}$ without $\Sigma_{1}$ induction. We show that a version of the Sacks Splitting Theorem for r.e. sets fails very badly in these models. In particular, we prove that there is a complete r.e. set for which the Sacks Splitting Theorem for r.e. sets does not apply. This leads to the natural question as to whether we also get a strong failure of degree splitting in these models (see question (4) at the end of the paper).

We assume that the reader is familiar with the basics of recursion theory on fragments of Peano arithmetic (cf. Mytilinaios and Slaman [12] for details). All models considered are models of $P^{-}+I \Sigma_{0}$. Henceforth, when we say that $\mathscr{M}$ is a model of $B \Sigma_{n}$ we will implicitly mean that $\mathscr{M}$ is a model of $P^{-}+I \Sigma_{0}+B \Sigma_{n}$. Let $\mathscr{M}$ be a model of $P^{-}+I \Sigma_{0}$. We let $W_{e}$ denote the $e$ th r.e. set, and $K_{c}$ the $c$ th $\mathscr{M}$-finite set (more specifically, $K_{c}=\left\{y \mid \exists z, r\left(c=2^{y+1} z+r\right) \& 2^{y+1}>r \geq\right.$ $\left.\left.2^{y}\right\}\right)$. The inclusion of exponentiation as a function symbol will then guarantee 
that every $\Sigma_{0}$ set will be coded (by the usual application of induction). $W_{e, s}$ means the $\mathscr{M}$-finite subset of $W_{e}$ enumerated after $s$ steps. $\Phi_{e}$ denotes the $e$ th reduction procedure, where $\Phi_{e}(A ; x)=B(x)$ means that there exist $K_{c} \subset A$ and $K_{d} \subset \bar{A}$ such that $(x, i, c, d) \in W_{e, s}$ and $B(x)=i . \quad \Phi_{e}(A)=B$ if $\Phi_{e}(A ; x)=B(x)$ for every $x$.

$B$ is said to be pointwise recursive in $A$ (written $B \leq_{p} A$ ) if $\Phi_{e}(A)=B$ for some $e$. The notation $\leq_{T}$ refers to Turing reducibility, in which $\mathscr{M}$-finite sets, rather than individual elements, are to be computed as being contained in the set or in its complement. When we speak of degrees we will always mean degrees under Turing reducibility. This notion is well defined in any model of $P^{-}+I \Sigma_{0}$ while the degree notion for pointwise reducibility even for r.e. sets is known to require $B \Sigma_{2}$. The problem is that pointwise reducibility may not be transitive (see Groszek and Slaman [9]). In all models of $P^{-}+I \Sigma_{0}$ it is easy to see that transitivity of Turing reducibility holds and in addition that if $A \geq_{T} C$ and $C \geq_{p} B$ then $A \geq_{p} B$ (again see [9] for details).

\section{A FRIEDBERG-MUCHNIK PAIR}

The first difficulty in any attempt to construct a Friedberg-Muchnik pair (i.e., two r.e. sets which are incomparable) in a model $\mathscr{M}$ (of, for example, $B \Sigma_{1}$ ) without $I \Sigma_{1}$ is the failure of the priority argument at the inductive stage. More precisely, $\Sigma_{1}$ induction is used in an essential way to prove that every requirement is satisfied. In its absence, there is no least counterexample and so proof by induction fails. Model-theoretically, this translates into the existence of a proper $\Sigma_{1}$ cut which witnesses the satisfaction of requirements. However, since this cut is proper (not the whole model) there will be requirements for which we will never have the opportunity to try and satisfy. Nevertheless, the usual. construction, being $\Delta_{0}$ (with exponentiation), is not affected in this restricted framework. See [8] for a discussion on recursion-theoretic strategies in relation to priority arguments.

This then leads one to consider other types of constructions of a different nature than the usual Friedberg-Muchnik type. It is here that we encounter a related second difficulty arising from the need to deal with nonamenable sets. A set $A$ is amenable if its intersection with every $\mathscr{M}$-finite set is $\mathscr{M}$-finite. Every r.e. set in a model of $\Sigma_{1}$ induction is amenable. However in the model studied by Slaman and Woodin [15], every amenable r.e. set is recursive, and all nonrecursive r.e. sets (necessarily nonamenable) have degree above the degree of the cut. (Chong and Mourad [2] actually showed that this set is of minimal degree.) In [10] it is shown that in all models of $B \Sigma_{1}$ which do not satisfy $I \Sigma_{1}$ we have all amenable sets either recursive or of the same degree as the $\Sigma_{1}$ cut. This means that in these models any Friedberg-Muchnik pair has to be a nonamenable pair as well.

The priority technique such as that used in the usual proof of the FriedbergMuchnik Theorem appears to yield little insight for the construction of incomparable nonamenable pairs, although in some cases this difficulty has been circumvented in higher recursion theory through the use of definable projecta having nice properties (for example, in the case where the $\Sigma_{1}$ projectum of an inadmissible ordinal is a regular cardinal). The basic problem with nonamenable r.e. sets is that the standard finite injury priority method fails, since 
negative conditions may be injured unboundedly many times, and so there is no stage after which a requirement is permanently satisfied. Indeed this suggests searching for a new approach which would attempt to turn this apparent obstruction into an aid for constructing nonamenable pairs. This is sometimes referred to as the 'double edged sword' approach to generalized recursion theory.

In particular, we could try to construct a Friedberg-Muchnik pair of nonamenable-sets by attempting to destroy computations threatening to make one side compute the other. Nonamenability would allow us to enumerate elements less than some bounded point in the model arbitrarily late in the construction. This is indeed the approach adopted here. To elaborate, let us introduce the notion of a Post style strategy.

Recall that in his attempt to find an r.e. degree which was neither complete nor recursive, Post considered r.e. sets whose complements were infinite yet very thin in that they did not contain much accessible 'information' which could be used in Turing reductions. This would presumably make them good candidates for being incomplete at the same time as being nonrecursive (since complements of recursive are in no sense thin, being themselves recursive). As is now known, this approach does not work. In fact, the constructions by Sacks and Yates of complete maximal sets showed that this approach could fail quite strongly. Let us analyze this situation in more detail.

In trying to build an r.e. set $A$ which does not compute some other r.e. set we may try to destroy any computation which attempts to use any information about $A$ that was not otherwise available. Since $A$ is r.e., such information would indicate which numbers are in the complement of $A$. The general strategy could then be to destroy these computations by enumerating the appropriate numbers in $A$. Now the problem here is that we may be forced to enumerate too many elements and make $A$ recursive. However, one may hope to be able to tell when such a situation arises and take alternative action. Depending on the context, this may mean putting an element on the other side or not putting any elements in and arguing that the set computed would be recursive. We will refer to this approach as a Post style strategy.

Now, in the classical case of $\omega$ it is not clear at any finite stage what it would mean to put in too many elements. A set with infinite complement may be very dense on its initial segments yet be recursive. Global properties of having thin complement do not correspond to local density considerations, and moreover, because of the Sacks-Yates result mentioned above, may be of no help in making the set incomplete.

Essentially, the reasons for this are related to $\omega$ being a regular cardinal. In contrast with $\omega$, models which do not satisfy $I \Sigma_{1}$ have a certain 'shape' which is reminiscent of singular cardinals. In the presence of such singularity, it is indeed possible to make the above strategy successful. Here the global structure of the model can be better approximated by local considerations. This is most naturally seen in the case of a singular cardinal where, as long as one does not fill up the gap between ordinals of successive cardinalities of the cofinal sequence, one will not put in too many elements. A computation procedure which would force us to fill (densely) such a gap would be seen to do so at a bounded stage by singularity considerations. If this is the case then as suggested above we take appropriate action at that stage to permanently satisfy the requirements.

Now this suggests that, locally, the structure of our set need not be complex. 
In fact in [3] we show that a union of intervals (as many as the cofinality) suffices for a successful application of a Post style strategy. In the case of arithmetic we consider the notion of a union of cuts. We discuss this in detail below.

A novelty of our approach is that the use of priority is generally avoided. Instead the strategy used can be simultaneously applied to a whole block of requirements with cardinality (pigeonhole principle) considerations ensuring compatibility. This is a crucial point, as the basic problem in such a setting where singularity plays such a strong role is that there are far fewer steps in the construction than there are requirements to be satisfied. In fact it should be remarked that König's theorem in cardinal arithmetic is in a sense an archetype for the following construction in the same sense as Cantor's diagonal argument is for the Friedberg-Muchnik argument. We also mention that some of the obstacles here are rather similar to those encountered in computational complexity theory and that some of these ideas may find application there.

The following is a standard result whose proof we omit.

Proposition. Let $\mathscr{M}$ be a model of $P^{-}+I \Sigma_{0}+\neg I \Sigma_{1}$. Then there is a cut $I$ and a $\Sigma_{1}$ function $f$ total on $I$, such that $\{f(i) \mid i \in I\}$ is cofinal in $\mathscr{M}$.

A cut $I$ satisfying the conclusion of the Proposition is called a $\Sigma_{1}$ cut.

We now state a lemma that will be crucial for applying the cardinality considerations indicated above.

$\Delta_{n}$ Pigeonhole Principle. Let $\mathscr{M}$ be a model of $B \Sigma_{n}$. Let $a, b \in \mathscr{M}$ and let I be a $\Sigma_{n}$ set which is bounded above. Also let $f$ be a $\Sigma_{n}$ function with domain $I$ which takes as its values $\mathscr{M}$-finite sets each of which has cardinality less than $a$. Let $X$ be contained in the union of the range of $f$. Then for some $b \in I$ :

(a) $X$ is contained in an $\mathscr{M}$-finite union of $b$ many such sets.

(b) $X$ cannot contain an $\mathscr{M}$-finite set of size $a b$.

Proof. Assume that $X$, an $\mathscr{M}$-finite is in (the union of) the image of $f$. Consider the function from each element $x$ of $X$ to the least $t$ witnessing that $x \in f(i)$ for some $i \in I$. By the definitions of $X$ and $f$ this function is $\Sigma_{n}$ and hence must have bounded image (by $B \Sigma_{n}$ ). Therefore, for some $b \in I, X$ is in the union of no more than $b$ many $\mathscr{M}$-finite sets of cardinality less than $a$. Then by $\Sigma_{0}$ induction (recall that all of our models satisfy $\left.I \Sigma_{0}\right) X$ has size less than $a b$.

Theorem 1 (The Friedberg-Muchnik Theorem). Let $\mathscr{M}$ be a model of $\mathrm{P}^{-}+$ $I \Sigma_{0}+B \Sigma_{1}$. Then there exist r.e. sets $A$ and $B$ such that neither is pointwise recursive in the other.

Proof. If $\mathscr{M}$ is a model of $I \Sigma_{1}$, then as discussed earlier the Friedberg-Muchnik Theorem already holds. Thus let us assume that $\mathscr{M}$ does not satisfy $I \Sigma_{1}$. Let $f$ be a definable strictly increasing function (over $\mathscr{M}$ ) whose domain is a cut. A set $A \subset \mathscr{M}$ is called a union of cuts if for each $i$ in the domain of $f$, $A \cap[f(i), f(i+1))$ is a cut $J$, i.e., $J$ is either all of $[f(i), f(i+1))$ or bounded below $f(i+1)$, has no maximum element, and is closed downwards when viewed within $[f(i), f(i+1)]$. The key idea of our solution lies in applying a Post style strategy to a union of cuts to define both sets with the pigeonhole principle playing the role that cardinality considerations play in the case of a singular cardinal. In fact we will consider a sequence of cuts where the size of each successive cardinal grows, as in the case of a singular cardinal. It should 
not be too surprising that such sets can act as a Friedberg-Muchnik pair, since Slaman and Woodin have shown that the cut is sufficiently complicated to act as a solution to Post's problem in any model of $B \Sigma_{1}$ but not of $I \Sigma_{1}$. The sets $A$ and $B$ will be constructed as unions of cuts.

Let $\mathscr{M}$ be a model with a $\Sigma_{1}$ cut $I$ and a $\Sigma_{1}$ function $f$ such that $f$ and $I$ satisfy the conditions of the Proposition. We may assume that for some $b$ an upper bound of $I, f(i+1)-f(i)>2^{b(f(i))^{3}}$ for each positive $i \in I$ (for a start, let us set $f(0)>b$ and $\left.f(1)-f(0)>2^{b(f(0))^{3}}\right)$. This is a technical device to ensure that each interval $[f(i), f(i+1)]$ is large enough to simultaneously accommodate the strategies in the diagonal argument attempting to satisfy requirements of the type $\Phi_{e}(A) \neq B\left(\right.$ and $\left.\Phi_{e}(B) \neq A\right)$ for $e<f(i)$. We call $(I, f, b)$ an admissible triple.

As we have previously remarked, the situation here is parallel to that of limit cardinals $\alpha$. In the latter case we have $\alpha$ a limit of regular cardinals, so that if $I$ is now the cofinality of $\alpha$ and $f$ its cofinality function which is strictly increasing and takes only successor cardinals as values, then the interval $[f(u), f(u+1))$, for $u \in I$, has cardinality $f(u+1)$ and any subset of $[f(u), f(u+1))$ of cardinality at most $f(u)$ is bounded below $f(u+1)$. This property is exploited in [3] to produce positive solutions to Post's problem.

Let $f, b$ be given so that $(I, f, b)$ forms an admissible triple. Let $A$ be a union of cuts. First of all, if $K_{c} \subset \bar{A}$, then for each $i \in I, K_{c} \cap[f(i), f(i+1))$ (if nonempty) is contained in an interval of the form $[x, f(i+1)$ ), where $x$ is the least element of $K_{c}$ in $[f(i), f(i+1))$. It is thus sufficient to deal only with $\mathscr{M}$-finite sets $K$ whose intersection with every interval $[f(i), f(i+1))$ consists of at most one point. Such sets are denoted $K^{*}$. There is an effective enumeration of such $\mathscr{M}$-finite sets (in fact this is a recursive collection), and we denote these as $\left\{K_{c}^{*} \mid c \in \mathscr{M}\right\}$. Secondly, if $A$ is an r.e. union of cuts, then one may assume, by introducing a new enumeration if necessary, that if $x \in[f(i), f(i+1))$ is enumerated in $A_{s}$, then every $y<x$ in $[f(i), f(i+1))$ is also in $A_{s}$. Finally, by a change of parameter if necessary, in dealing with requirements of the form $\Phi_{e}(A) \neq B$, it is enough to handle only negative conditions. Namely, one needs to consider only triples of the form $(x, 0, c) \in$ $W_{e}$ or $(x, 1, c) \in W_{e}$, where $K_{c}^{*} \subset \bar{A}$. The reason here being that if we have $(x, 0, c, d) \in W_{e}$ or $(x, 1, c, d) \in W_{e}$, with $K_{c} \subset A$ and $K_{d} \subset \bar{A}$, then if we set $g\left(K_{c}, j\right)$ to be the maximum of members of $K_{c}$ in $[f(j), f(j+1))$, we have, by the fact that $I$ is a $\Sigma_{1}$ cut, that there is a stage $s$ where each $g\left(K_{c}, j\right)$ is enumerated in $A_{s}$. By the second observation, this implies that $K_{c} \subset A_{s}$. Now by going to a different $e$ (in an effective manner), this positive condition can be suppressed.

We begin our construction with the empty set, and then declare that for each $i \in I, A \cap[f(2 i), f(2 i+1))=[f(2 i), f(2 i+1))$ and $B \cap[f(2 i+1), f(2 i+2))=$ $[f(2 i+1), f(2 i+2))$. This will give us $A_{0}$ and $B_{0}$. For $j$ odd, let $a_{j, 0}=f(j)$ and $\bar{a}_{j, 0}=f(j+1)-1$. Define $b_{j, 0}, \bar{b}_{j, 0}$ similarly for $j$ even.

Assume that $s \in I$ is even, $A_{s}, B_{s}$ have been constructed, and that each of $A_{s} \cap[f(2 i+1), f(2 i+2)), B_{s} \cap[f(2 i), f(2 i+1))$ is of the form $[f(2 i+1), r]$, $[f(2 i), t]$ respectively. For $j<s+1$ odd, let $a_{j, s}$ be the largest element of $[f(j), f(j+1))$ which is in $A_{s}$, and (by construction) let $\bar{a}_{j, s}$ be the least element in the interval already declared to be in $\bar{A}$ (forever). 
Given $K^{*}$, denote the least element of $\left[K^{*} \cup\{f(j+1)\}\right] \cap[f(j), f(j+1)]$ by $d\left(K^{*}, j\right)$. Assume by induction that $\bar{a}_{j, s}-a_{j, s}>2^{(b-s) f(j)^{3}}$. Define $b_{k, s}, \bar{b}_{k, s}$ similarly for $k$ even. Fix $i$ to be odd. In what follows, we adopt the convention that $j>i$ denotes an odd number, while $k$ denotes an even number. Let $(\alpha, \beta, \gamma)=\alpha f(i+2)^{2}+\beta f(i+2)+\gamma$. For each $e<f(i)$ and each $u<2^{f(i+1)}$ such that $K_{u}^{*} \subset \bar{A}_{s} \cap[0, f(i+1))$ let

$$
\begin{aligned}
& D_{u, e, s+1}=\left\{(c, x) \mid(x, 0, c) \in W_{e, f(s+1)} \& K_{u}^{*} \subset K_{c}^{*}\right. \\
& \left.\quad \& \forall j>i\left[d\left(K_{c}^{*}, j\right)>a_{j, s}\right] \& \bar{b}_{i+1, s} \geq x>b_{i+1, s}\right\} .
\end{aligned}
$$

Step 1: In increasing order of $r=(u, e, x)$, where $x \in[f(i+1), f(i+2))$, we will define $a_{j, s+1}^{r}$ and $\bar{a}_{j, s+1}^{r}$ inductively. Setting $a_{j, s+1}^{0}=a_{j, s}$ and $\bar{a}_{j, s+1}^{0}=$ $\bar{a}_{j, s}$, and assuming $a_{j, s+1}^{r-1}$ and $\bar{a}_{j, s+1}^{r-1}$ have been defined for some $r>0$, see if there is a $c \leq f(s+1)$ such that $(c, x) \in D_{u, e, s+1}$ and

$$
\forall j>i\left[d\left(K_{c}^{*}, j\right)-a_{j, s+1}^{r-1}>2^{(b-s)(f(j))^{3}-r}\right] .
$$

If such a $c$ exists, let $c(u, e, x)$ denote the least such $c$. Let $\bar{a}_{j, s+1}^{r}=$ $d\left(K_{c(u, e, x)}^{*}, j\right)$ and let $a_{j, s+1}^{r}=a_{j, s+1}^{r-1}$.

At this point, we are insuring that we will have a large enough interval to accommodate similar action at subsequent stages in the construction. If such a $c$ exists then we can have a potential computation which will cause us to enumerate too many elements to destroy. In that case we preserve this computation. If a $c$ does not exist then we can destroy all potential computations as follows.

First let $\bar{a}_{j, s+1}^{r}=\bar{a}_{j, s+1}^{r-1}$. Now, we have for all $c \leq f(s+1)$, if $(c, x) \in$ $D_{u, e, s}$, then there is a $j>i$ such that

$$
d\left(K_{c}^{*}, j\right)-a_{j, s+1}^{r-1} \leq 2^{(b-s)(f(j))^{3}-r} .
$$

For each $c$ such that $(c, x) \in D_{u, e, s+1}$, we destroy its computation by enumerating all $z$ in $A$, with $a_{j, s+1}^{r-1}<z \leq a_{j, s+1}^{r-1}+2^{(b-s)(f(j))^{3}-r}$ for each $i<j<s+1$. Therefore let $a_{j, s+1}^{r}=a_{j, s+1}^{r-1}+2^{(b-s)(f(j))^{3}-r}$ for all such $j$.

Observe that after $(u, e, x)$ is taken care of, we have

$$
\bar{a}_{j, s+1}^{r}-a_{j, s+1}^{r}>2^{(b-s)(f(j))^{3}-r} .
$$

Set

$$
a_{j, s+1}=\operatorname{Max}\left\{a_{j, s+1}^{r} \mid e<f(i), u<2^{f(i+1)}, x \in\left(b_{i+1, s}, \bar{b}_{i+1, s}\right]\right\}
$$

and

$$
\bar{a}_{j, s+1}=\operatorname{Min}\left\{\bar{a}_{j, s+1}^{r} \mid e<f(i), u<2^{f(i+1)}, x \in\left(b_{i+1, s}, \bar{b}_{i+1, s}\right]\right\} .
$$

Since $u<2^{f(i+1)}, e<f(i)$, and $x \in\left(b_{i+1, s}, \bar{b}_{i+1, s}\right)$, there are at most $2^{f(i+1)}$. $f(i) \cdot f(i+2)$ many triples $(u, e, x)$. Since $2^{f(i+1)}<f(i+2)$, we see that $(f(i+2))^{3}$ is an upper bound for the codes of these triples. Since $j>i$ is odd (so $j$ is at least $i+2)$, we have for $j>i, \bar{a}_{j, s+1}-a_{j, s+1}>2^{(b-(s+1))(f(j))^{3}}$. This insures that irrespective of what action we take we will always have enough elements left to work with in each interval on the ' $A$ ' side. 
Step 2: We now make sure that we enumerate elements in $B$ for which we could not destroy all computations without enumerating too many elements. Our Post style strategy will now succeed because in this case we preserve at least one disagreement having taken the appropriate action for $A$ above. Let $T=\left(b_{i+1, s}, \bar{b}_{i+1, s}\right)$. For each $e<f(i)$ and $u<f(i+1)$, if there is an $x \in T$ such that $c(u, e, x)$ exists, let $x_{u, e}$ be the least $x$ corresponding to $c(u, e, x)$. Then the set $\left\{x_{u, e} \mid e<f(i), u<f(i+1)\right\}$ partitions $T$ into at most $f(i)$. $f(i+1)+1$ many parts. Since $\bar{b}_{i+1, s}-b_{i+1, s}>2^{(b-s)(f(i+1))^{3}}$, there is at least one part of the partition having length at least $2^{(b-(s+1))(f(i+1))^{3}}$. Choose the least such part, and denote it by $\left[x_{u, e}, x_{v, r}\right]$. Let $b_{i+1, s+1}=x_{u, e}$ and $\bar{b}_{i+1, s+1}=x_{v, r}$. Enumerate all $z, b_{i+1, s}<z \leq x_{u, e}$, in $B$.

This ends the construction at stage $s+1$. At stage $s+2$, we interchange the roles of $A$ and $B$ in the construction, and interchange the parities of $i$, $j$, and $k$. Let $A=\bigcup_{s \in I} A_{s}$ and $B=\bigcup_{s \in I} B_{s}$. Note that $A$ and $B$ are unions of cuts. Let $t<b$ be an upper bound of $I$. Now for all $s$, and for all $i$ and $j>i, \bar{a}_{j, s}-a_{j, s}>2^{(b-s)(f(j))^{3}}>2^{(b-t)(f(j))^{3}}$ and $\bar{b}_{i+1, s}-b_{i+1, s}>$ $2^{(b-s)(f(i+1))^{3}}>2^{(b-t)(f(i+1))^{3}}$. This implies that for each $j$, the two sequences $\left\{a_{j, s}\right\}$ and $\left\{\bar{a}_{j, s}\right\}$ are respectively nondecreasing and nonincreasing. We claim that there exist $z$ 's which lie between $\bar{a}_{j, s}$ and $a_{j, s}$ for every $s \in I$. This can be shown by considering the sets

$$
\mathscr{J}_{s}=\left\{y \mid a_{j, s}<y \leq a_{j, s+1}\right\} \cup\left\{y \mid \bar{a}_{j, s}>y \geq \bar{a}_{j, s+1}\right\}
$$

for each $s \in I$. If we consider the function which takes $s \in I$ to $\mathscr{J}_{s}$ then, using the fact that $\bar{a}_{j, s}>a_{j, s}$ for all $s \in I$, a direct application of the $\Delta_{1}$ Pigeonhole Principle (a) (see above) shows that $\bigcup \mathscr{J}_{s}$ is not the whole interval $[f(j), f(j+1))$, giving us our required $z$ 's. The same argument applies for the ' $B$ ' side.

We verify that $A$ and $B$ form a Friedberg-Muchnik pair. Suppose that $\Phi_{e}(A)=B$. Choose $i$ such that $f(i)>e$ ( $i$ odd), and let $x \notin B$ such that $b_{i+1, s}<x<\bar{b}_{i+1, s}$ for every $s \in I$. This $x$ exists as shown above by the $\Delta_{1}$ Pigeonhole Principle (a). If there is a $c$ such that $K_{c}^{*} \subset \bar{A},(c, x) \in D_{u, e, s}$ for some $u$ and $s$, and (1) holds at stage $s+1$, then $x_{u, e}$ is defined, and if, on the one hand, we have $x_{u, e} \leq b_{i+1, s+1}$, so that $x_{e} \in B$, then $\Phi_{e}\left(A ; x_{u, e}\right)=$ $0 \neq B\left(x_{u, e}\right)=1$, contradicting the fact that $\Phi_{e}(A)=B$. This is the case since there is a number $c$ such that for all $j>i, \bar{a}_{j, s+1} \leq d\left(K_{c\left(u, e, x_{u, e}\right)}^{*}\right)$ (therefore $\left.K_{c\left(u, e, x_{u, e}\right)}^{*} \subset \bar{A}\right)$. If, on the other hand, we have $\bar{b}_{j, s+1} \leq x_{u, e}$ this contradicts the fact that $x_{u, e} \leq x<\bar{b}_{j, s+1}$.

Now suppose that a $c$ satisfying (1) does not exist at any stage. Fix a $c$ so that $K_{c}^{*} \subset \bar{A}$, and $(c, x) \in D_{u, e, s+1}$ for some $u$ and $s$. Then (2) holds for some $j>i$. Now in Step 1 of the construction we set $a_{j, s+1}^{r} \geq d\left(K_{c}^{*}, j\right)$ and so $d\left(K_{c}^{*}, j\right)$ is enumerated in $A$. This contradicts the assumption that $K_{c}^{*} \subset \bar{A}$. Thus $\Phi_{e}(A) \neq B$.

Recall that the Sacks Splitting Theorem states that every nonrecursive r.e. set can be split into two sets of strictly lower degree whose union is the original set. A weaker version of this statement says that every nonrecursive r.e. degree can be split into two strictly lower degrees. We refer to this result as the Sacks Splitting Theorem for r.e. degrees. We will shortly conclude from Theorem 1 
that even this weaker statement is strictly stronger than the existence of two incomparable r.e. degrees. We now state a result from [10] which will allow us to compare the proof-theoretic strength of the Friedberg-Muchnik Theorem with that of the Sacks Splitting Theorem. We will also use this result in $\S 3$.

Definition. A $\Sigma_{n}$ degree, $\mathbf{b}$, is said to be splitting iff there exist $\Sigma_{n}$ degrees $\mathbf{d}_{1}$ and $d_{2}$ such that $\mathbf{d}_{1} \vee \mathbf{d}_{2}=\mathbf{b}, \mathbf{d}_{1} \neq \mathbf{b}$, and $\mathbf{d}_{2} \neq \mathbf{b}$.

Theorem 2. Let $\mathscr{M}$ be a model of $P^{-}+I \Sigma_{0}+B \Sigma_{n}+\neg I \Sigma_{n}$. Let I denote a $\Sigma_{n}$ cut in such a model. Let $A$ be a $\Sigma_{n}$ set. Then either $A$ is $\Delta_{n}$ or $A \geq_{T} I$. In particular, the degree of $I$ is not a splitting degree and if $A$ is nonamenable then $A \geq_{T} I$. Therefore the degree of $I$ is least among the $\Sigma_{n} \backslash \Delta_{n}$ degrees.

This shows that over the base theory $P^{-}+I \Sigma_{0}+B \Sigma_{1}$ the Sacks Splitting Theorem for r.e. degrees implies $I \Sigma_{1}$. On the other hand, Mytilinaios [11] has shown that the full Sacks Splitting Theorem (for r.e. sets) can be proven in $P^{-}+I \Sigma_{1}$.

Corollary 1. The Friedberg-Muchnik Theorem is strictly weaker than the Sacks Splitting Theorem for r.e. degrees.

Proof. As discussed above, the Sacks Splitting Theorem is equivalent to $I \Sigma_{1}$ over $P^{-}+I \Sigma_{0}+B \Sigma_{1}$. In fact Theorem 2 shows that there exists a least nonrecursive r.e. degree in models of $P^{-}+I \Sigma_{0}+B \Sigma_{1}+\neg I \Sigma_{1}$. Therefore we have that the Friedberg-Muchnik Theorem holds in these models (by Theorem 1) while the Sacks Splitting Theorem for r.e. degrees fails.

The techniques introduced for Theorem 1 have found applications in ordinal recursion theory. As previously mentioned, in [3] we show that there is a positive solution to Post's problem above $\varnothing^{\prime}$ for all admissible ordinals, under the notion of dynamic reducibility (in contrast with Friedman's negative result for $\aleph_{\omega_{1}}^{L}$ under Turing reducibility [6]). A positive solution to Post's problem under dynamic reducibility is also found for all inadmissible ordinals. A further refinement of this technique is used to show in [4] that for $\aleph_{\omega}^{L}$, there is a positive solution to Post's problem between $\varnothing^{\prime}$ and $\varnothing^{\prime \prime}$, under Turing reducibility.

\section{INCOMPARABLE $\Sigma_{n}$ SETS}

Just as in the situation where $n=1$, sets above a $\Sigma_{n}$ cut (i.e., those which compute a $\Sigma_{n}$ cut ) are by necessity nonamenable, and therefore present us with the same obstacles as before. But here we have an additional twist: the cofinal function on a $\Sigma_{n}$ cut is now $\Sigma_{n}$, rather than $\Sigma_{1}$ (i.e., recursive). However, this function may be chosen so that its graph is Turing reducible to $\varnothing^{(n-1)}$. Now using $\varnothing^{(n-1)}$ as an oracle, one can imitate the construction given in Theorem 1, relativized to $\varnothing^{(n-1)}$, to produce a Friedberg-Muchnik pair $\Sigma_{n}$ above $\varnothing^{(n-1)}$. We briefly sketch how the argument goes: Let $\mathscr{M}$ be a model of $P^{-}+B \Sigma_{n}+$ $\neg I \Sigma_{n}$. Fix a $\Sigma_{n}$ cut $I$ in $\mathscr{M}$, a $\Sigma_{n}$ increasing cofinal function $f$ total on $I$, a $b$ which is an upper bound of $I$, and assume that $(I, f, b)$ is an admissible triple. To define $\Sigma_{n} \backslash \Delta_{n}$ sets $A$ and $B$, first let $A \cap\{(x, 0) \mid x \in \mathscr{M}\}=B \cap\{(x, 0) \mid x \in$ $\mathscr{M}\}=\left\{(x, 0) \mid x \in \varnothing^{(n-1)}\right\}$. This insures that $\varnothing^{(n-1)} \leq_{T} A$ and $\varnothing^{(n-1)} \leq_{T} B$. Now follow the construction in Theorem 1 , recursively in $\varnothing^{(n-1)}$, to obtain a Friedberg-Muchnik pair $A$ and $B$ whose members are $\Sigma_{1}$ in $\varnothing^{(n-1)}$, and so 
$\Sigma_{n}$. Furthermore, the sets $A$ and $B$ can be made to be $\Delta_{n}$ incomparable since we can replace functionals given by $W_{e}$ by those given by $W_{e}^{\varnothing^{(n-1)}}$.

In [2] we introduced a technique to show that for $n \geq 2$, the sets $I$ (a $\Sigma_{n}$ cut) and $\varnothing^{(n-1)}$ form a minimal pair in a given model $\mathscr{M}$ of $P^{-}+I \Sigma_{0}+B \Sigma_{n}+\neg I \Sigma_{n}$. In the case where every $\Delta_{n+1}$ subset of $I$ is coded on $I$ (i.e., when it is the intersection of an $\mathscr{M}$-finite set with $I$ ), it is even true that $I$ is of minimal degree. The technique involves an analysis of how sets pointwise recursive in $I$ are computed, via the notion of recoverability and truth sets. In this section we will discuss some results from [5] which use a variant of this technique to study sets which lie above $\Sigma_{n}$ cuts. Crucial to the application of these ideas to all models of $B \Sigma_{n}$ is the following Coding Lemma. This lemma is also crucial to the results quoted from [10] and we will later make use of it in $\S 3$.

Definition. Let $\mathscr{M}$ be a model of $P^{-}+I \Sigma_{0}$. Given sets $X$ and $Y$ we say $X$ is coded on $Y$ iff there exists an $\mathscr{M}$-finite set $Z$ such that $Z \cap Y=X \cap Y$.

Definition. Let $\mathscr{M}$ be a model of $P^{-}+I \Sigma_{0}$. Given sets $X$ and $A$ we say that $X$ is $\Delta_{n, A}$ iff both $X \cap A$ and $\bar{X} \cap A$ are $\Sigma_{n}$.

Coding Lemma. Let $\mathscr{M}$ be a model of $P^{-}+I \Sigma_{0}+B \Sigma_{n}$. Then every $\Delta_{n, A}$ set, $X$, is coded on $A$.

Proof. Assume this is false. Consider the function taking a potential code for $X$ to an element witnessing that this potential code is not correct. If all approximations to the required code are eventually seen to be false, then by $B \Sigma_{n}$ this will be witnessed at a fixed bounded point for all such approximations, but we can always define better approximations even if we can not define the actual code, a contradiction. For full details see [10] or [2].

A key result of [5] is the following:

Theorem 3. Let $\mathscr{M}$ be a model of $P^{-}+I \Sigma_{0}+B \Sigma_{n}+\neg I \Sigma_{n}$, where $n \geq 2$. Let $I$ be a $\Sigma_{n}$ cut. Then there exists a class of amenable sets, $\Theta$, containing the $\Delta_{2}$ sets such that the map

$$
A \mapsto A \oplus I
$$

for $A \in \Theta$, is order-preserving on pointwise and Turing reducibility. Moreover, for sets $A, B \in \Theta$,

$$
A \oplus I \leq_{T} B \oplus I \leftrightarrow A \leq_{T} B \leftrightarrow A \leq_{p} B \leftrightarrow A \oplus I \leq_{p} B \oplus I .
$$

Remark. Theorem 3 applies as well to $\Delta_{1}$ sets when $n=1$, although in this case the result does not provide any useful information since all $\Delta_{1}$ sets occupy the same degree.

The Friedberg-Muchnik Theorem for $\Sigma_{n}$ sets for $n \geq 2$ can now be given for sets whose degrees are above the degree of $\varnothing^{(n-1)} \oplus I$ and also for those whose degrees are above the degree of $I$ but below the degree of $\varnothing^{(n-1)} \oplus I$. In the latter case one can even choose sets whose degrees are splitting.

Corollary 2. Let $\mathscr{M}$ be a model of $P^{-}+I \Sigma_{0}+B \Sigma_{n}$. Then there exist two $\Sigma_{n} \backslash \Delta_{n}$ sets $A$ and $B$, whose degrees are above the degree of $\varnothing^{(n-1)} \oplus I$, which form $a$ Friedberg-Muchnik pair, i.e., $A \not_{p} B$ and $B \not_{p} A$, and two $\Sigma_{n} \backslash \Delta_{n}$ sets $A$ and $B$ whose degrees are below the degree of $\varnothing^{(n-1)} \oplus I$ yet above the degree of $I$ 
which also form a Friedberg-Muchnik pair. In the latter case $A$ and $B$ can be chosen so that the degree of each is splitting.

Proof. For the first claim the construction indicated in the beginning of this section gives the sets required. Note that these sets are not $\Delta_{n}$ having degrees strictly above the degree of $\varnothing^{(n-1)}$ and hence also strictly above the degree of $I$ by Theorem 2 .

For the other claim, let $A$ and $B$ be $\Delta_{2}$ sets such that neither is pointwise reducible to the other (take, for example, a Friedberg-Muchnik pair for r.e. sets). Let $I$ be a $\Sigma_{n}$ cut and by Theorem 3, we have $A \oplus I$ and $B \oplus I$ to be incomparable, $\Sigma_{n}$, and not $\Delta_{n}$. Since $\mathscr{M}$ satisfies $I \Sigma_{1}$ we can choose sets whose degrees are splitting by the previously quoted result due to Mytilinaios [11]. It then follows from Theorem 3 that the corresponding degrees induced by the map $A \mapsto A \oplus I$ are also splitting. Note that in these cases the degrees of these sets cannot be above the degree of $\varnothing^{(n-1)}$ since otherwise they would be equal to the degree of $\varnothing^{(n-1)} \oplus I$, which is impossible because they are incomparable.

We refer the reader to the questions at the end of the paper concerning splitting degrees above the degree of $\varnothing^{(n-1)} \oplus I$. The general technique and topic treated in this section may be developed and expanded further with applications to priority arguments. This will be taken up in [5] and in future papers.

\section{SACKS SPLitTING FOR R.E. SETS}

We will now show that a certain version of Sacks splitting fails in a strong way in models of $B \Sigma_{1}$ without $\Sigma_{1}$ induction. We will give examples of complete r.e. sets which cannot be split into two smaller Turing degrees. We will also provide a wide array of r.e. sets which we cannot split into pointwise incomparable sets, also giving strong restrictions on the subsequent Turing degrees. This still leaves open the possibility that a weak form of splitting is available for some sets and degrees (see the questions at the end of the paper).

Theorem 4. Let $\mathscr{M}$ be a model of $P^{-}+I \Sigma_{0}+B \Sigma_{1}+\neg I \Sigma_{1}$. If $A$ is an r.e. set, then there exist r.e. sets $B$ and $C$ such that

(a) $A \leq_{T} B, C \leq_{T} A$ (hence $C \leq_{T} B$ ), $B \leq_{p} C$, and $B \leq_{p} A$.

(b) $C$ is a union of cuts.

(c) If $B=B_{1} \cup B_{2}\left(B_{1} ; B_{2}\right.$ r.e. $)$, then $B_{k} \leq_{p} A, B, C$ for $k=1,2$ and $\exists k \quad\left(k=1\right.$ or $k=2$ ) so that $B_{k} \geq_{T} A$ (hence $\left.B_{k} \geq_{T} C\right), B_{k} \geq_{p} B$, and $B_{k} \geq_{p} B_{3-k}$.

(d) If $A$ is complete then $A \equiv_{T} B$ and hence in (c) $B_{k} \equiv_{T} B$ and $B_{k} \geq_{T}$ $B_{k-3}$.

Proof. First note that (d) follows immediately from (a), (c), and the fact that $A$ is complete. Let $(I, f, b)$ be an admissible triple. Given an $\mathscr{M}$-finite set $K$, let $[K]$ denote the code of $K$. Let

$$
B=\left\{b_{i, j} \mid i, j \in I ; \forall i \geq j\left(b_{i, j}=\left[A_{f(i)} \mid f(j)\right]\right) \& \forall i<j\left(b_{i, j}=2^{f(j-1)}\right)\right\} .
$$

Without loss of generality, we may assume that $f(i) \in A_{f(i+1)}$ for each $i \in I$. (We can always find a Turing equivalent $A$ with this property). This insures that $2^{f(j-1)} \leq b_{i, j}<2^{f(j)}$ for all $i, j$ (note that for all $\mathscr{M}$-finite $K \subset f(j)$, $[K] \leq 2^{f(j)}<f(j+1)$ since we have chosen an admissible triple $\left.(I, f, b)\right)$. Now let

$$
C=\left\{x \mid \exists i, j\left(b_{0, j} \leq x \leq b_{i, j}\right)\right\} .
$$


Note that $C$ is just obtained from the set $B$, closing downwards within each interval $[f(j), f(j+1)]$. We first make one observation about this coding which we will use later on. We note that if one set contains another then the code of the first set will be greater than or equal to that of the second one. From this (and $B \Sigma_{1}$ ) it follows that if $x$ is such that there exists a $j$ such that for all $i \in I, b_{i, j}<x<b_{0, j+1}\left(=2^{f(j)}\right)$ then there exists an $\mathscr{M}$-finite set $X$ such that $[X]=x$ and $X \cap \bar{A} \mid f(j) \neq \varnothing$. (We use the above remark on coding and the fact that an $\mathscr{M}$-finite set $T$ satisfies $T \subset A$ if and only if $T \subset A_{f(i)}$ for some $i \in I$ (by $\left.B \Sigma_{1}\right)$.)

We first show that $B \geq_{T} A$. Since all sets considered here are r.e. and we are working in models of $B \Sigma_{1}$, we need only deal with complements to prove that a set computes another. Now,

$$
\begin{aligned}
T \subseteq \bar{A} \leftrightarrow \exists j \forall x \in T(x<f(j) \& P=\{[K] \mid K \mathscr{M} \text {-finite, }, & K \cap T \neq \varnothing \\
& K \subset \mathscr{M} \mid f(j)\} \subset \bar{B}) .
\end{aligned}
$$

Observe that $P$ is $\mathscr{M}$-finite. Conversely, for pointwise reducibility we have

$$
\begin{aligned}
x \in \bar{B} \leftrightarrow \exists K \exists y[([K]=x \& y \in K \cap \bar{A}) & \\
\vee & \left.\exists i \exists j\left(K \subset A_{f(i)}\right) \mid f(j) \& \forall t \leq i \forall u \leq j\left(x \neq b_{t, u}\right)\right],
\end{aligned}
$$

showing that $B \leq_{p} A$. We we will also be able to conclude this independently from the following:

Note that for each $j$, the set $\left\{b_{i, j} \mid i \in I\right\}$ is a nondecreasing sequence, so that if $b_{i, j}<x<b_{i+1, j}$, then $x \notin B$. Hence

$$
x \notin B \leftrightarrow \exists j\left(x<b_{0, j} \&\left\{y \mid x \leq y<b_{0, j}\right\} \subset \bar{C} \vee \exists i\left(b_{i, j}<x<b_{i+1, j}\right)\right) .
$$

This gives us $B \leq_{p} C$. Fix some $j \in I$ and consider $A \mid f(j)$. Now consider the function $h_{j}$ which takes $i \in I$ to $b_{i, j}$. It follows that there is an $\mathscr{M}$-finite set which codes an extension of $h_{j}$ with domain $\leq d$, where $d \notin I$, such that the extension has the property that its image codes a nested sequence of $\mathscr{M}$-finite subsets of $\mathscr{M} \mid f(j)$. This can be easily deduced from the Coding Lemma of $\S 2$ above. (For complete details see [10].) Call such a code $K_{j}$. Now consider the following reduction:

$$
\begin{aligned}
T \subseteq \bar{C} \leftrightarrow \exists j \exists S[\forall x \in T(x<f(j)) \& S \mathscr{M} \text {-finite } \& S \subseteq \bar{A} \& \\
\forall y \in T \exists \hat{j} \leq j\left(y<b_{0, \hat{j}} \&\left[\forall z \left(y \leq z<b_{0, \hat{j}}\right.\right.\right. \\
\rightarrow \exists Z([Z]=z \& Z \cap S \neq \varnothing)])] .
\end{aligned}
$$

We must verify that this is correct. It is easy to see that any $T$ satisfying the right-hand side must be a subset of $\bar{C}$ by the fact that $C$ is the downward closure of elements coding subsets of $A$. On the other hand, assume that for some $T$ such that $T \subseteq \bar{C}(T, \mathscr{M}$-finite) there exist no such $S$ as above, allowing $A$ to compute that $T \subseteq \bar{C}$. Now let $j$ be such that $f(j)$ is an upper bound for $T$. Consider $K_{j}$ as defined above. For each $l \leq d$ consider the set $V_{l}$ which is such that $\left[V_{l}\right]=K_{j}(l)$. If $l \notin I$ then $A \mid f(j) \subseteq V_{l}$ and hence $\bar{V}_{l} \subseteq \bar{A}$. Now, each set $S=\bar{V}_{i}$ for $i \in I$ is such that it satisfies the bottom condition of $(*)$ since $S \supseteq \bar{A} \mid f(j)$ (by the earlier remarks on coding). Then by overspill there must be some $\bar{V}_{j}$ with $j \notin I$ which satisfies both the top and bottom condition of $(*)$, and hence gives us the required $S$. This gives us 
$C \leq_{T} A$. We can then conclude from the transitivity of Turing computability that $C \leq_{T} B$ and (independently of the above) $B \leq_{p} A$. Thus (a) and (b) are established.

We now prove (c). Suppose that $B$ is split into the union of r.e. sets $B_{1}$ and $B_{2}$. We first show $C \geq_{p} B_{k}$ for $k=1,2$. It will then follow from (a) that we have $A \geq_{p} B_{k}$ and $B \geq_{p} B_{k}$ for $k=1,2$.

$$
\begin{aligned}
x \notin B_{k} \leftrightarrow \exists j\left(x<b_{0, j} \&\left\{y \mid x \leq y<b_{0, j}\right\} \subset \bar{C}\right. & \\
\qquad & \left.\exists i\left(b_{i, j}<x<b_{i+1, j}\right) \vee x \in B_{k-3}\right) .
\end{aligned}
$$

To show the rest of $(\mathrm{c})$ let

$$
X_{k}=\left\{(i, j)\left|B_{k, f(i)}\right|\left[2^{f(j-1)}, 2^{f(j)}\right) \neq B_{k, f(i+1)} \mid\left[2^{f(j-1)}, 2^{f(j)}\right)\right\},
$$

where $B_{k, f(i)}$ means computing $B_{k} f(i)$ many steps and $2^{f(-1)}$ denotes the number 0 . Then $X_{k} \subset I \times I$. We say that $X_{k}$ is eventually bounded if there is a $j_{0}$ such that for all $j>j_{0}, Y_{k, j}=\left\{i \mid(i, j) \in X_{k}\right\}$ is bounded in $I$.

Case 1. For each $k, X_{k}$ is eventually bounded. This means that $B_{k}$ is amenable above some $f(j)$. We claim that $I \geq_{T} B_{k}$ for each $k$. Now the set $X_{k}$ is $\Delta_{1, I \times I}$. So by the Coding Lemma it is coded on $I \times I$ by an $\mathscr{M}$-finite set $\hat{X}_{k}$. Let $j_{0} \in I$ be chosen so that for all $j>j_{0}, Y_{k, j}$ is bounded in $I$. Let $B_{k}$ be written as $\left\{w_{i, j} \mid i \in I \& 2^{f(j-1)} \leq w_{i, j}<2^{f(j)}\right\}$. Let $R_{k}=\left\{\left(i, j, w_{i, j}\right) \mid i \in I \& j \leq j_{0}\right\}$ and let $G=I \times I \times\left\{v \mid v \leq f\left(j_{0}\right)\right\}$. Then $R_{k}$ is $\Delta_{1, G}$ and so again by the Coding Lemma is coded on $G$ by an $\mathscr{M}$-finite set $\widehat{R}_{k}$. One may now compute $B_{k}$ from $I$ as follows:

Fix $a$ an upper bound of $I$. Given $T \subseteq\left[0,2^{f(j)}\right.$ ), let $H$ be the set of all $i$ 's such that $(i, j) \in \widehat{X}_{k} . H$ is $\mathscr{M}$-finite. Now see if $j>j_{0}$. If the answer is yes, we have

$$
T \cap B_{k} \neq \varnothing \leftrightarrow \exists s, t \leq a \exists i \leq s\left(s \in I \& t \notin I \& H \cap[s, t]=\varnothing \& w_{i, j} \in T\right) .
$$

Otherwise, we have $j \leq j_{0}$ and

$$
\begin{aligned}
T \cap B_{k} \neq \varnothing \leftrightarrow \exists i \leq a[ & \exists x\left((i, j, x) \in \widehat{R}_{k} \& x \in T\right) \\
& \left.\& \forall i^{*}<i \forall x\left(x \in T \rightarrow\left(i^{*}, j, x\right) \notin \widehat{R}_{k}\right) \& i \in I\right] .
\end{aligned}
$$

Then $I \geq_{T} B_{k}$, and so $I \geq_{T} B, A, C$. By Theorem 2 it follows that each of $B, B_{1}, B_{2}, A$, and $C$ is either of degree $I$ or is recursive. Therefore we must have $B_{k} \equiv_{T} B \equiv_{T} A \equiv_{T} C$ for some $k$ by part (a).

Case 2. $X_{1}$ (say) is not eventually bounded. Let $U$ be the set of all $j$ 's such that $Y_{1, j}$ is bounded in $I$. If $U$ is bounded by $i_{0} \in I$, we claim that $A \leq_{T} B_{1}$ as follows. Given an $\mathscr{M}$-finite set $T$, we first find $r \in I, r>i_{0}$, so that $T \subset[0, f(r)]$. Now consider

$$
Z_{T}=\left\{\left[T^{*}\right] \mid T^{*} \subset[0, f(r)] \& T \cap T^{*} \neq \varnothing\right\} .
$$

Then $T \subset \bar{A}$ if and only if $Z_{T} \subset \bar{B}_{1}$. Thus $A \leq_{T} B_{1}$.

Now if $U$ is unbounded, then we show $A \leq_{T} B_{2}$ as follows: Since $U$ is unbounded, it means that for unboundedly many $j \in I$, the set $Y_{2, j}$ will be unbounded since in this case (Case 2) $B$ is not amenable.

Let $\widehat{X}_{1}$ be as defined in Case 1. Since $B_{2}$ is not amenable, we have $B_{2} \geq_{T} I$ (by Theorem 2 ). Compute $A$ just as before using $Z_{Y}$, but now we must find an 
$r \in I$ which, in addition to all the other properties, also satisfies the following: There exist $t \in I$ and $u \notin I$ so that for all $i \in(t, u],(i, r) \notin \widehat{X}_{1}$. Such $t$ and $u$ must exist by overspill since if $(i, r) \notin \widehat{X}_{1}$ for large enough $i$, then this must also hold up to some $u \notin I$. Since $B_{2} \geq_{T} I, B_{2}$ can compute such an $r$. Now for this $r$ we have $Y_{2, r}$ unbounded in $I$. This gives us the required reduction procedure (arguing $T \subset \bar{A} \leftrightarrow Z_{T} \subset \bar{B}_{2}$ ). Therefore we also have $B_{1} \geq_{T} C$ and $B_{1} \geq_{p} B$ (by $\left.(\mathrm{a})\right)$ and $B_{1} \geq_{p} B_{2}$ (since $C \geq_{p} B_{2}$ ).

We end this paper with several questions.

(1) What is the ultimate axiom system necessary and sufficient for the Friedberg-Muchnik Theorem to hold? In particular, is $P^{-}+I \Sigma_{0}$ (with exponentiation) sufficient?

(2) Let $\mathscr{M}^{*}$ be the model considered by Slaman and Woodin in [15]. In this model, every real is the standard part of an $\mathscr{M}$-finite set. In $\mathscr{M}^{*}$, the set $\omega$ is an r.e. set of minimal degree. What other properties does this set have? For example, is $\omega$ the infimum of two incomparable r.e. sets (in terms of pointwise or Turing reducibility)? Is $\omega$ incomparable with any definable set (in [2], we show that $\omega$ is comparable with any $\Sigma_{2}$ set)?

(3) In a model of $P^{-}+I \Sigma_{0}+B \Sigma_{1}+\neg I \Sigma_{1}$, is there an analog of the Friedberg Completeness Theorem (dealing with, say, sets rather than degrees)? For this problem to make sense in our weak setting, a proper choice of the notion of the jump of a set has to be made. Observe, however, that the classical proof uses finite forcing which yields a 1-generic set in the process. There are models of $B \Sigma_{1}$ in which 1-generic sets are known not to exist (see $\left.[4,5]\right)$. Thus again a different approach is called for.

(4) Is every degree strictly above the degree of $\varnothing^{(n-1)} \oplus I$ splitting in models of $B \Sigma_{n}$ ? We know this is false for the degree of the cut by Theorem 2, and Theorem 4 tells us that there are complete r.e. sets for which there is no splitting into two sets of smaller degree. However one may still be able to split the complete r.e. degree by finding one complete r.e. set which splits into two sets of smaller degrees. Is it possible to split such a set into two sets which are pointwise incomparable in addition to being Turing incomparable?

(5) Theorem 4 tells us that for every r.e. set $A$ there is an r.e. set $B$, which has greater Turing degree yet which $A$ computes pointwise, such that this set does not split into two pointwise incomparable sets. Can a set $A$ be constructed (necessarily not complete or of the same degree as that of the cut) such that the corresponding $B$ splits into two sets of strictly lesser Turing degree? This would also give us $A \searrow_{T} B$ by Theorem 4(c). Note that since the set $B$ depends on the enumeration of $A$ this would not be too surprising.

(6) Is the set $C$ corresponding to $A$ in Theorem 4(d) also complete? It computes pointwise the complete sets $A$ and $B$ yet we do not believe that it itself is complete since so much of its information is seemingly destroyed by downward closure.

\section{REFERENCES}

1. C. T. Chong, Recursively enumerable sets in models of $B \Sigma_{2}$, Mathematical Logic and Its Applications, Lecture Notes in Math., vol. 1388, Springer-Verlag, 1989.

2. C. T. Chong and K. J. Mourad, The degree of a $\Sigma_{n}$ cut, Ann. Pure Appl. Logic 48 (1990), $227-235$. 
3. __ Positive solutions to Post's problem, Recursion Theory Week, Lecture Notes in Math., vol. 1432, Springer-Verlag, 1990.

4. __ Post's problem and singularity (in preparation).

5. $\ldots, \Sigma_{n}$ cuts in models without $\Sigma_{n}$ induction (in preparation).

6. S. D. Friedman, Negative solutions to Post's problem, Ann. of Math. (2) 113 (1981), 25-43.

7. _,$\beta$ recursion theory, Trans. Amer. Math. Soc. 235 (1979), 173-200.

8. M. J. Groszek and T. A. Slaman, Foundations of the priority method I: Finite and infinite injury (to appear).

9. __ On Turing reducibility (to appear).

10. K. J. Mourad, The Sacks Splitting Theorem and $\Sigma_{1}$ induction (to appear).

11. M. Mytilinaios, Finite injury and $\Sigma_{1}$ induction, J. Symbolic Logic 54 (1989), 38-49.

12. M. Mytilinaios and T. A. Slaman, $\Sigma_{2}$ collection and the infinite injury priority method, J. Symbolic Logic 53 (1988), 212-221.

13. J. B. Paris and L. A. Kirby, $\Sigma_{n}$ collection schemas in models of arithmetic, Logic Colloquium '77, North-Holland, 1978.

14. G. E. Sacks and S. G. Simpson, The $\alpha$-finite injury method, Ann. Math. Logic 4 (1972).

15. T. A. Slaman and W. H. Woodin, $\Sigma_{1}$ collection and the finite injury priority method, Mathematical Logic and Its Applications, Lecture Notes in Math., vol. 1388, Springer-Verlag, 1989.

Department of Mathematics, National University of Singapore, Singapore 0511

Current address, K. J. Mourad: Department of Mathematics, Ohio State University, Columbus, Ohio 43210

E-mail address, C. T. Chong: scicct@nuscc.nus.sg 